\title{
BIBECHANA
}

\section{Rotaxane: a switching element in molecular electronics}

\author{
N. K. Chaudhary* \\ Dept. of Chemistry, M.M.A.M. campus Biratnagar, Nepal \\ Article History: Received 5 October 2010; Revised 15 November 2010; Accepted 22 November 2010
}

\begin{abstract}
The switching in materials is the most interesting phenomenon discovered in materials, both organic and inorganic. The switching materials opened a large class of applications in electronics and informatics. Rotaxane is one such type of molecule which shows switching ability by the action of external stimuli either chemical or physical. Reversible structural change occurring in rotaxane due to external stimulus is described and its application in nanorecording is also studied.
\end{abstract}

Keywords: CBPQT (cyclobis(paraquat-p-phenylene); TTF (tetrathiafulvalene); DNP (1,5dioxynaphthalene\}

\section{Introduction}

Rotaxanes have novel characteristics as a switching element in the molecular electronics. With the development of supramolecular chemistry, it has become possible to synthesize rotaxanes by template directed synthesis method developed by Leigh et al. Switching action of rotaxanes is due to movement of macrocycle over the axis of dumbbell shaped molecules. This opened a large class of applications in electronics and informatics. The use of switching in computer memories, in information recording and the application in automation and robotics are due to polyfunctional activities of rotaxanes.

\section{Structure of Rotaxane}

A rotaxane is a mechanically interlocked molecular architecture consisting of a dumbbell shaped molecule, that is threaded through a macrocycle or ring-like molecule. Its key structure contains a pie $(\pi)$ electron deficient macrocycle that is locked around a pie $(\pi)$ electron rich component along the molecular thread with two bulky stoppers .The macrocycle can move between different $\operatorname{pie}(\pi)$ electron rich components of dumb bell shaped molecule by the action of external stimuli either chemical or physical, resulting in switching of electronic configuration [1]. This creates new configuration of molecules with different functions and acts as molecular

\footnotetext{
* Corresponding author: N.K. Chaudhary, Department of Chemistry, M.M.A.M. Campus, Tribhuvan

University, Biratnagar, Email: chem_narendra@yahoo.com
} 
switch. The binding site of macrocycle can be reversed back to its original position by another stimulus. Such a molecule constitutes molecular shuttle.

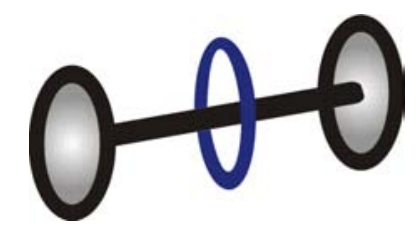

Graphical representation of a rotaxane

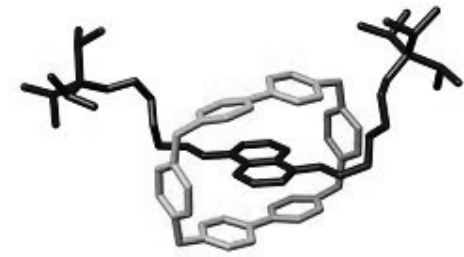

Cyclobis(paraquat-p-phenylene)

A Rotaxane molecule

Firure: 1

External stimulus applied to affect in switching motion of rotaxane weakens the binding forces between the macrocycle and dumbbell shaped molecule. Macroscopic machine uses power for intercomponental relative position changes while a switchable rotaxane performs shuttling motion in response to external stimulus. So molecular shuttle is also called molecular machine.

\subsection{Applications}

\section{A. In molecular electronics as a memory device}

Due to ability of rotaxanes to exist between two different co-conformations, they can store high density mass data irreversibly and show nonvolatile memory effect $[2,3]$. Controlling switching motion of rotaxane in solid state could be used to store information by encoding the two different states into binary digits.

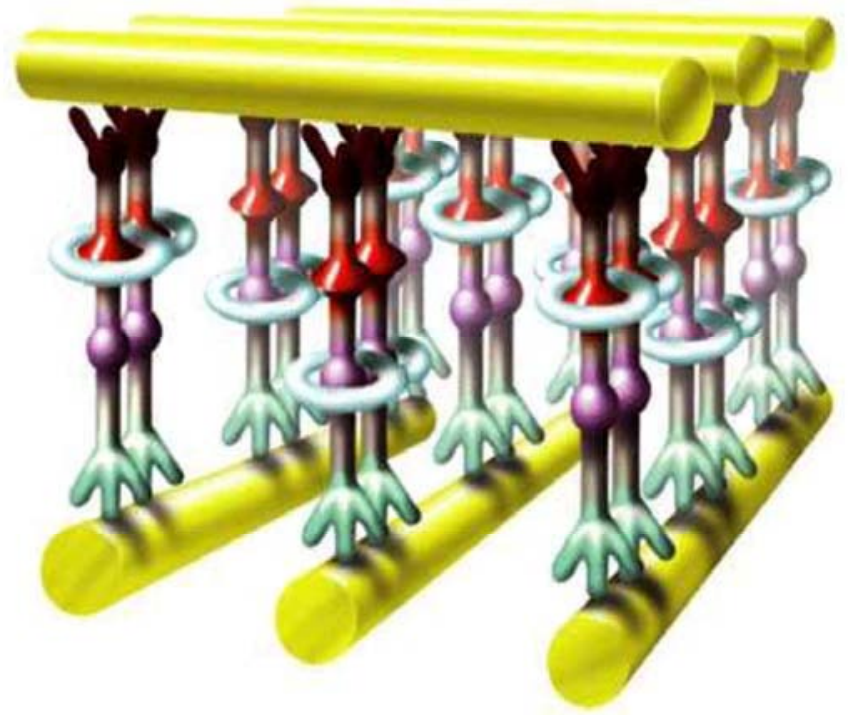

Figure 2: Graphical representation of a molecular memory based on [2] Rotaxane. 
In a nanorecording application, a certain rotaxane is deposited as a Langmuir -Blodgett film on ITO coated glass. When a +ve voltage is applied with a tip of scanning tunneling microscope probe, the rotaxane rings in the tip area switch to a different part of dumbbell and the resulting new conformation makes the molecules stick out from the surface by $0.3 \mathrm{~nm}$ [4] and this height difference turns out to be sufficient for a memory dot. The dots could encode data into binary digits and such a film has capacity to store data up to 100 Gbits per square inch.

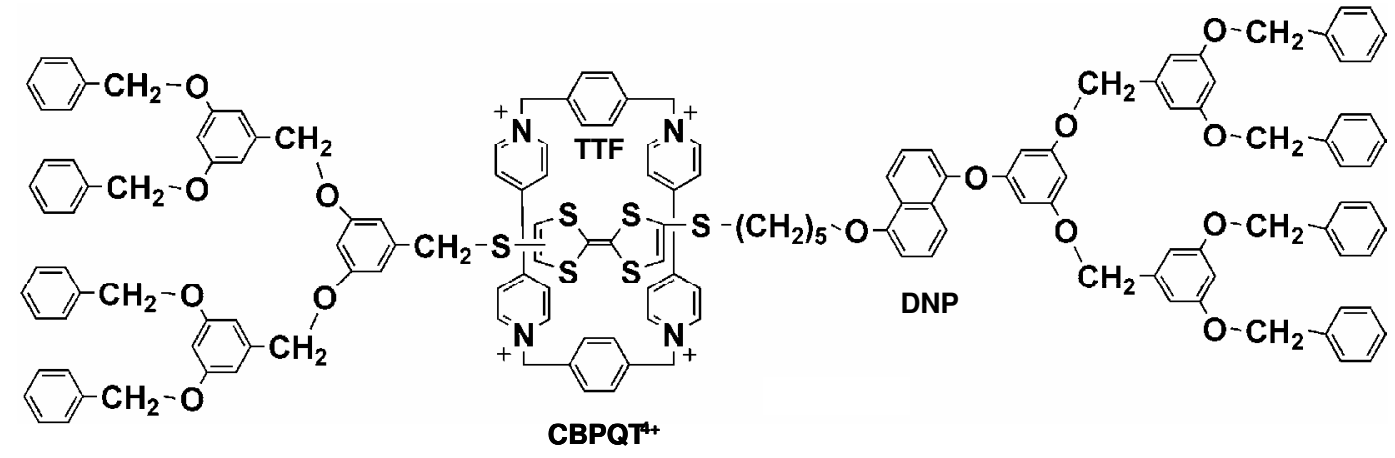

Figure 3: The switchable [2] Rotaxane used for nonorecording.

In the bistable rotaxane[2] shown above that consists of a pie-acceptor tetracationic ring (CBPQT ${ }^{4+}$ ) and two pie-donor stations (TTF and DNP), switching is originated due to reversible mechanical movement of the CBPQT ${ }^{4+}$ macrocycle between TTF and DNP component triggered by chemical stimuli or electrical stimulus. In the ground state of the switch [5], the paraquat ring is located around a tetrathiafulvalene (TTF) unit but it moves to the dioxynapthyl unit (DNP) when the fulvalene unit is oxidized by application of a current.

\section{B. As a molecular transistor}

Another use of switchable rotaxanes is for the fabrication of molecular transistors. A molecular shuttle can serve as a molecular switch, in that it can be triggered to switch between two different co-conformations. In the following scheme present an example of single molecule devices which contains molecular transistors based on a bistable [2] rotaxane. 
(a)

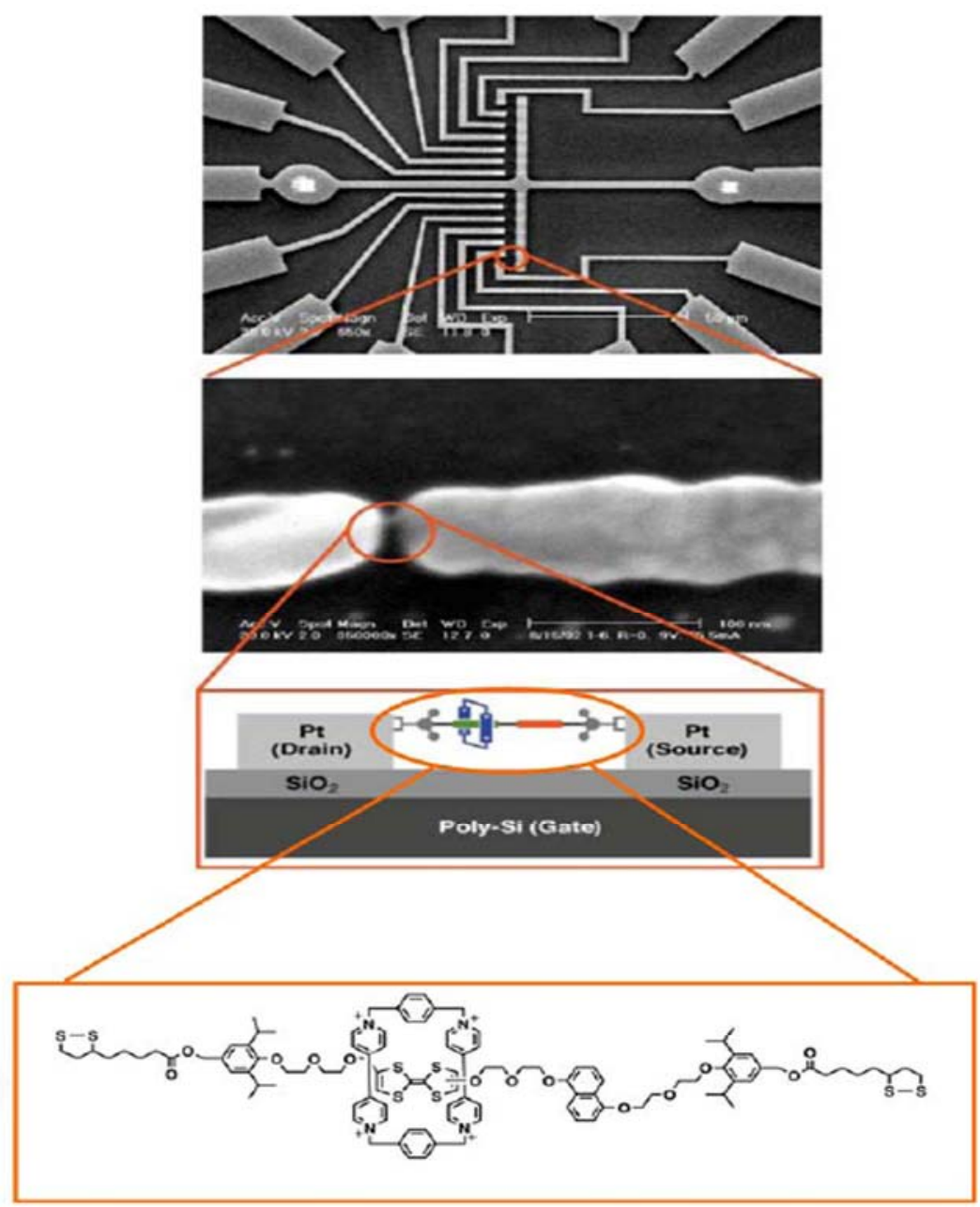

(b)

Figure 4: (a) SEM pictures of single molecular devices fabricated on a Si substrate. The centre metal electrode, where the break junction is made of $7 \mathrm{~nm}$ thick Pt, followed by $100 \mathrm{~nm}$ thick gold wires and pads for wire bonding.

(b) The SEM image after the break junction is made. The gap between the two metal electrodes is around $4 \mathrm{~nm}$.

(c) A cross section through a single molecule transistor.

(d) Rotaxane which the molecular transistor is based on [2].

\section{Conclusion}

Exploring the physical properties of different types of rotaxanes on solid surfaces provides versatile and valuable information for the development of atomic and molecular devices and will open new future world in molecular electronics. It is inspiring that more fantastic properties have been found for nanometer scale materials, and more novel methods have been developed for fabricating nanometer scale functional materials. However, towards practical 
application, there are still many challenges. We expect that these goals can be achieved by the collaboration of specialists in different areas and by the successful integration of various techniques into the challenging research on atomic scale engineering.

\section{References}

[1] M. Feng, X. Guo, X. Lin, X. He, W. Ji, S. Du, D. Zhang, D. Zhu, and H.Gao J. Am. Chem. Soc., 127 (2005)15338.

[2] H. Tian and Q. C. Wang, Recent progress on switchable rotaxanes, Chem. Soc. Rev., 35 (2006)361.

[3] J. P. Miron (PhD Thesis-2008), Universitat Autonoma de Barcelona -Molecular modeling of switchable[2] rotaxanes.

[4] M. Popescu, A. Velea, A. Lőrinczi, F. Sava, J. Ovonic Research 5 (2009) 27.

[5] H. J. Gao, Li Gao, Progress in Surface Science, 85 (2010) 28. 